Please do not remove this page

RMIT

UNIVERSITY

\title{
Significant Enhancement of Antimicrobial Activity in Oxygen-Deficient Zinc Oxide Nanowires
}

Elbourne, Aaron; Cheeseman, Samuel; Wainer, Pierce; Kim, Jaewon; Medvedev, Alexander; Boyce, Kylie; McConville, Chris

https://researchrepository.rmit.edu.au/esploro/outputs/9921887134901341/filesAndLinks?institution=61RMIT_INST\&index=null

Elbourne, A., Cheeseman, S., Wainer, P., Kim, J., Medvedev, A., Boyce, K., McConville, C., van Embden, J., Crawford, R., Chapman, J., Truong, V. K., \& Della Gaspera, E. (2020). Significant Enhancement of Antimicrobial Activity in Oxygen-Deficient Zinc Oxide Nanowires. ACS Applied Bio Materials, 3(5), 2997-3004. https://doi.org/10.1021/acsabm.0c00065

Document Version: Accepted Manuscript

Published Version: https://doi.org/10.1021/acsabm.0c00065

Repository homepage: https://researchrepository.rmit.edu.au

(c) 2020 American Chemical Society

Downloaded On 2023/04/26 12:17:52 +1000 


\section{Significant Enhancement of Antimicrobial Activity in Oxygen-Deficient Zinc Oxide Nanowires}

Aaron Elbourne ${ }^{1}$, Samuel Cheeseman ${ }^{1}$, Pierce Wainer ${ }^{1}$, Jaewon Kim ${ }^{1}$, Alexander E. Medvedev ${ }^{2}$,

Kylie. J. Boyce ${ }^{3}$, Christopher F. McConville ${ }^{1}$, Joel van Embden ${ }^{1}$, Russell J. Crawford ${ }^{1}$, James

Chapman $^{1}$, Vi Khanh Truong ${ }^{1, *}$,Enrico Della Gaspera ${ }^{1, *}$

${ }^{1}$ School of Science, RMIT University, Melbourne VIC 3001, Australia.

${ }^{2}$ School of Engineering, RMIT University, Melbourne, Victoria 3001, Australia.

${ }^{3}$ School of Science, RMIT University, Bundoora, VIC 3083, Australia.

KEYWORDS ZnO; nanostructures; oxygen vacancies; antibacterial; chemical bath deposition 


\section{ABSTRACT}

The fabrication of antimicrobial surfaces that exhibit enhanced activity towards a large variety of microbial species is one of the major challenges of our time. In fact, the negative effects associated with both bacterial and fungal infections are enormous, especially considering that many microbial species are developing resistance to known antibiotics. In this work, we show how a combination of specific surface morphology and surface chemistry can create a surface that exhibits nearly $100 \%$ antimicrobial activity towards both Gram-negative and Gram-positive bacteria, and fungal cells. Arrays of vertically aligned, oxygen deficient zinc oxide $(\mathrm{ZnO})$ nanowires grown on a substrate exhibit enhanced antimicrobial activity compared to surfaces containing either less defective nanowires, or highly oxygen-deficient flat films. This synergistic effect between physical activity (morphology) and chemical activity (surface composition) has been shown to be responsible for the outstanding antimicrobial activity of our surfaces, especially towards notoriously resilient bacterial or fungal species. These findings provide a series of design rules for tuning the activities of antibacterial and antifungal nanomaterials. These rules constitute an excellent platform for the development of next-generation antimicrobial surfaces. 


\section{INTRODUCTION}

Pathogenic microbial surface colonization, commonly referred to as biofilm formation, represents a significant healthcare and industrial concern. This is particularly true for surfaces where sterilization is required, such as those present on medical implants, hospital surfaces, and other medical devices. This issue is further impacted by the current rise in antibiotic resistance amongst microbial species, which has caused a significant increase in post-operative infections and related deaths in recent years. ${ }^{1,2,3,4}$ Economic projections have estimated that bacterial infections could be responsible for upwards of 10 million deaths per annum by 2050 if new antibacterial therapies are not developed. ${ }^{5}$ In addition to bacteria, fungal cells are also estimated to infect 1.7 billion people annually, resulting in $\sim 1.5$ million deaths per annum, with these figures also increasing in recent years. ${ }^{6,7}$ Despite these staggering figures, the contribution of fungal infections to the global burden of disease remains poorly recognized.

In response to these issues, considerable research has focused on the development of surfaces that are capable of mitigating both bacterial and fungal biofilm formation. The two main mitigation strategies rely on either the addition of antimicrobial or inhibitory agents - including nanoparticles, 8 antibiotics, ${ }^{9}$ and polymers ${ }^{10,11}$ - to the outer surface of biocompatible materials (chemical activity), or to the micro/nanostructuring of surfaces in order to achieve mechanical rupturing of the cell membranes when coming into contact with the nanofeatures, implying a physical killactivity. ${ }^{12,13}$ Pure chemical approaches have become less viable as a long-term, antibacterial solution due to instances of increasing antibiotic resistance, ${ }^{14}$ toxicity concerns about nanomaterials, ${ }^{15}$ and dosage complications. ${ }^{16}$ Antibacterial nanostructures that are effective as a result of the physical effects of the surface are widely found in nature, such as the nanopillar arrays 
native to cicada wings, and the hierarchically structured, self-cleaning lotus leaves. These natural surfaces have led to the development of numerous biomimetic, synthetic counterparts. ${ }^{13,17,18}$ However, they are intrinsically non-selective and their activity is heavily dependent on both the type of microorganism and the specific surface geometry present on the substrate surface. ${ }^{12,17}$ Combining both physical and chemical activity is therefore key to producing highly efficient antimicrobial surfaces, given the interplay of many factors, including bacterial cell size and physiology, the underlying topographical dimensions, specific bacterial adsorption processes, surface chemistry and the hydrophobicity/hydrophilicity of the surface. ${ }^{18}$

In this regard, chemical synthesis of (nano)materials is a versatile and powerful means for the development of materials and surfaces with the desired structure, morphology and chemical composition. ${ }^{19,20,21}$ As such, chemical methods have been extensively used to fabricate materials for applications such as optics, electronics, biology, catalysis, sensing, energy generation and storage. $^{22,23,24,25}$ One of the main advantages of chemical methods for the synthesis of nanomaterials is the ability to precisely tune the composition of the target material. In fact, both doping and off-stoichiometric compositions in inorganic nanomaterials have been widely adopted in order to tune their properties and improve device performances..$^{26,27,28,29}$ In particular, oxygen vacancies in metal oxides have been extensively studied and recently exploited to harness enhanced optical, electrical, electrochemical and catalytic properties in the oxygen-deficient materials compared to the stoichiometric oxide counterparts. ${ }^{21,30,31,32,33}$

In this work, we show the combined effect of surface morphology and oxygen deficiency in the antimicrobial activity of zinc oxide $(\mathrm{ZnO})$ nanostructures against both bacterial and fungal species. Highly textured $\mathrm{ZnO}$ nanowire "forests" provide an ideal surface morphology that physically limits the proliferation of microorganisms (physical activity). Their performance is greatly 
enhanced, however, when these oxide surfaces are made highly oxygen deficient (chemical activity). Importantly, highly oxygen deficient flat $\mathrm{ZnO}$ surfaces show reduced activity, confirming the synergistic effect between chemical and physical microbiocidal activity. The results reported herein afford further insights into the role of both chemical and physical contributions in nanostructured surfaces for inducing microbial cell death. The findings of this research highlight the importance of non-stoichiometry in the antimicrobial activity of metal oxide nanostructures and could potentially be extended to other oxide materials of interest. All this combined will aid in the development of next-generation surfaces that can prevent surface microbial colonization.

\section{RESULTS AND DISCUSSION}

$\mathrm{ZnO}$ nanowires (NWs) supported on glass and silicon substrates were grown using a modified chemical bath deposition (CBD) approach as described previously. ${ }^{30}$ Full experimental details are reported in the Methods section. Briefly, after depositing a ZnO seed layer using conventional solgel chemistry, the seeded substrates were dipped in a solution containing zinc nitrate and ammonia and heated to $80^{\circ} \mathrm{C}$ to trigger $\mathrm{ZnO}$ growth. This method enables the growth of uniform arrays of $\mathrm{ZnO}$ NWs, highly oriented along the $c$-axis of the hexagonal wurtzite cell. To obtain flat $\mathrm{ZnO}$ surfaces, ammonium citrate was added. Citrate anions are known to bind preferentially to polar surfaces of $\mathrm{ZnO}$ crystals, either $\mathrm{Zn}$-terminated (0001) or O-terminated (000-1) surface, therefore limiting the growth along the $<001>$ crystallographic orientation. This promotes "lateral" over "longitudinal" growth of the nanowires, resulting in dense films composed of columnar grains oriented along the $c$-axis. ${ }^{34}$ These dense $\mathrm{ZnO}$ films serve as a control surface for microbial testing, providing a surface with identical chemical composition, but with a rather different morphology. 

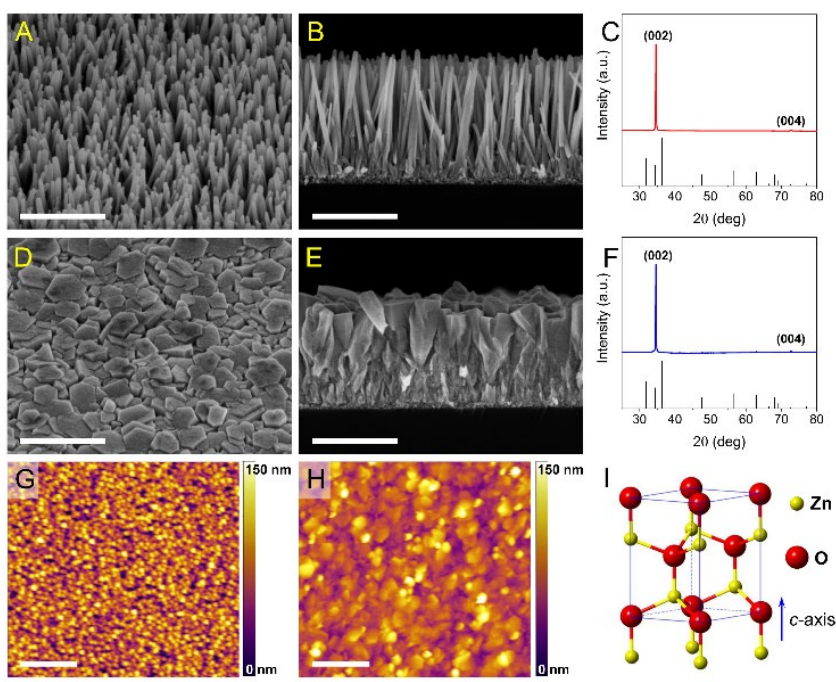

Figure 1. Morphological and structural characterization of the $\mathrm{ZnO}$ surfaces. SEM images in top view (45 degree tilt) and in cross section of $\mathrm{ZnO} N W$ arrays $(\mathrm{A}, \mathrm{B})$ and dense films $(\mathrm{D}, \mathrm{E})$. Scale bars are $500 \mathrm{~nm}$. The respective XRD patterns and the comparison with the predicted peak positions for bulk ZnO (ICDD No. 36-1451) are shown in panels (C) and (F). AFM profiles of ZnO NW arrays $(\mathrm{G})$ and dense films $(\mathrm{H})$. Scale bars are $1 \mu \mathrm{m}$. I) Schematic representation of the $\mathrm{ZnO}$ wurtzite crystalline cell.

Scanning electron microscopy (SEM) images of the resulting surfaces are shown in Figure 1 and Figure S1. Highly oriented $\mathrm{ZnO}$ wires can be clearly seen both in the top view and cross-sectional images. Notably, the simple addition of citrate causes a dramatic change in morphology, with the production of much larger wires that eventually merge together, resulting in a dense film.

The crystallographic orientation of these films has been demonstrated through X-ray diffraction (XRD), as shown in Figure 1C and 1F. All samples show prominent (002) diffraction peaks (at $2 \theta$ $=34.5^{\circ}$ ), and almost complete absence of any other peaks, with the exception of the higher order diffraction from the same family of lattice planes (i.e. $(004)$ at $\left.2 \theta=72.6^{\circ}\right)$. This evidence confirms the formation of highly textured $\mathrm{ZnO}$ crystals along the $c$-axis of the wurtzite cell. Atomic force 
microscopy (AFM) measurements were also employed to better determine the nano-architectures present on the substrate surfaces (see Figure $1 \mathrm{G}$, and $1 \mathrm{H}$ ). The topographical parameters of fabricated nanowire arrays and the flat $\mathrm{ZnO}$ films evaluated from SEM and AFM images are given in Table S1.

Importantly, this modified $\mathrm{CBD}$ enables the fabrication of non-stoichiometric $\mathrm{ZnO}$, and specifically highly oxygen deficient at the surface. This is verified with XPS through a detailed analysis of the $\mathrm{O} 1 \mathrm{~s}$ core-level peak. It is well established that the $\mathrm{O} 1 \mathrm{~s}$ peak can be fitted with three main components, corresponding to (from lower to higher binding energies) $\mathrm{O}^{2-}$ ions within the $\mathrm{ZnO}$ lattice ( $\mathrm{O}_{\text {I }}$ component), oxygen atoms residing in an oxygen-deficient environment or surface hydroxides $\left(\mathrm{O}_{\text {II }}\right.$ component) and to adsorbed oxygen-containing organic species $\left(\mathrm{O}_{\text {III }}\right.$ component). ${ }^{35} \mathrm{The} \mathrm{ZnO}$ nanowires synthesized in this study show an intense $\mathrm{O}_{\text {II }}$ component, with an integrated $\mathrm{O}_{\mathrm{I}} / \mathrm{O}_{\text {II }}$ signal of $\sim 1.2$, suggesting highly oxygen deficient nanowires (Figure $2 \mathrm{~A}$ ). Similarly, the dense films are also highly oxygen deficient (Figure S2). This enables the comparison of morphological effects (wires $v s$. dense films) whilst keeping the chemical activity similar. However, if the nanowires are annealed in air, the oxygen vacancies are progressively filled, resulting in more stoichiometric $\mathrm{ZnO}$ (Figure 2B). Notably, the morphology of the annealed nanowires is identical to that of the as-deposited materials (see Figure S3). This enables a direct comparison of the chemical effect (oxygen vacancies) in coatings possessing the same morphology. It is important to highlight that the $\mathrm{Zn} 2 \mathrm{p}$ signals of the samples are identical, confirming that the only change in the chemical nature of the $\mathrm{ZnO}$ surface is the presence of oxygen vacancies (see Figure S4). 

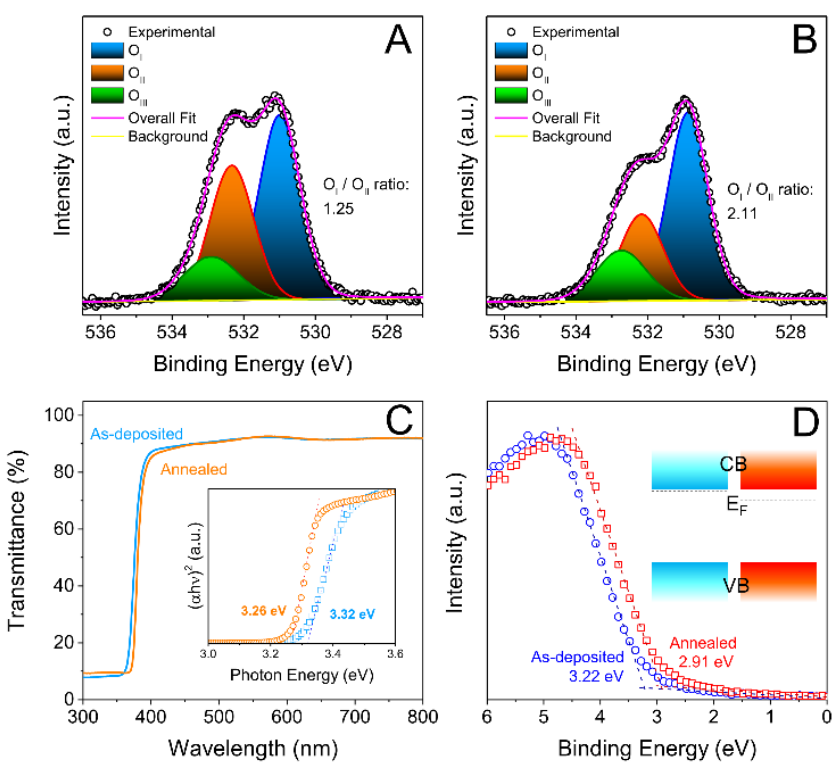

Figure 2. Optical and electronic characterization of ZnO NWs. X-ray photoelectron spectra of the $\mathrm{O} 1 \mathrm{~s}$ region for $(\mathrm{A})$ as-deposited and $(\mathrm{B})$ annealed $\mathrm{ZnO} \mathrm{NW}$ s showing the fitting with the three components. C) Transmittance spectra of as-deposited and annealed ZnO NWs. The inset shows the respective Tauc plot and band gap estimation. D) Valence band XPS for as-deposited and annealed $\mathrm{ZnO}$ NWs. The inset shows simplified band structures highlighting the downward shift in Fermi energy following annealing

The oxygen deficient nature of these $\mathrm{ZnO}$ was further proved through optical and electrical properties. The samples show a distinctive UV absorption onset at around $3.3 \mathrm{eV}$ typical of $\mathrm{ZnO}$. Close inspection of this region shows a small red shift of the band gap of 50-100 meV following annealing (Figure 2C). Given that the $\mathrm{ZnO}$ crystals are far beyond the quantum confinement regime, this shift could be ascribed to the Burstein-Moss effect. In fact, as-deposited $\mathrm{ZnO}$ is naturally oxygen deficient, and therefore has excess free electrons, similar to what is observed in aliovalently doped $\mathrm{ZnO} .{ }^{36,37}$ After annealing, the oxygen vacancies are at least partially filled, 
causing a reduction of the charge carrier concentration, and a reversal of the Burstein-Moss effect. Valence band (VB) XPS is used to evaluate the position of the Fermi energy $\left(\mathrm{E}_{\mathrm{F}}\right)$ with respect to the VB. Compared to as-deposited samples, the Fermi energy in annealed sample is located further $(\sim 0.3 \mathrm{eV})$ from the conduction band edge (Figure 2D). This is an indication of the reduced $n$-type character of annealed samples that can again be ascribed to a filling of the oxygen vacancies. This is further verified by electrical measurements on the dense films, which provide a sheet resistance of $\sim 20 \mathrm{k} \Omega / \square$ for unannealed $\mathrm{ZnO}$, and $\sim 700 \mathrm{k} \Omega / \square$ after annealing. The increase in resistance by over one order of magnitude confirms the reduction in charge carrier concentration subsequent to the filling of oxygen vacancies.

Having analyzed the morphology, composition and electronic structure of the $\mathrm{ZnO}$ coatings, we can now assess their antimicrobial performance. Escherichia coli (E. coli), methicillin-resistant Staphylococcus aureus (MRSA), and fluconazole-resistant Cryptococcus neoformans $\left(\right.$ fluc $^{\mathrm{R}}$ C. neoformans) cells were chosen as representative Gram-negative bacterium, Gram-positive bacterium, and yeast-like fungus, respectively. In this way different classes of single-celled pathogens could be tested, two of which (MRSA and C. neoformans) show resistance to conventional antibiotics. Representative confocal laser scanning microscopy (CLSM) images of the cells adsorbed on various $\mathrm{ZnO}$ surfaces, as well as on glass controls are shown in Figure 3a (see Materials and Methods section for details on the samples preparation). The number of live/dead cells were counted, evaluated as a percentage, and displayed as a bar chart. The corresponding values and their associated errors are also reported in the SI as Table S2 for easy comparison.

As-deposited $\mathrm{ZnO}$ NWs result in the highest amount of non-viable (dead) cells, specifically $95.3 \% \pm 4.3 \%, 68.8 \% \pm 10.5 \%$ and be $94.8 \% \pm 3.5 \%$ for Gram-negative E. coli, Gram-positive 
MRSA, and C. neoformans, respectively. Physically identical surfaces, but with reduced oxygen vacancy concentration, are obtained after annealing the $\mathrm{ZnO}$ NWs in air as discussed earlier. These annealed surfaces show a largely reduced antimicrobial activity, inactivating only $32.0 \% \pm 8.5 \%$, $19.5 \% \pm 1.9 \%$ and $16.7 \% \pm 4.5 \%$ of the E. coli, MRSA, and C. neoformans cells, respectively. Similarly, highly oxygen deficient flat surfaces (obtained introducing citrate as ligand during growth of $\mathrm{ZnO}$ ) also show a strongly reduced activity compared to the textured NW counterparts. In details, the inactivated cells on oxygen-deficient, flat $\mathrm{ZnO}$ for E. coli, MRSA, and $C$. neoformans are $22.3 \% \pm 7.6 \%, 27.9 \% \pm 10.8 \%$, and $3.3 \% \pm 1.2 \%$, respectively. These numbers are slightly higher than the values obtained for our control surfaces (plain glass slides), which show $<10 \%$ dead cells for all three species investigated.

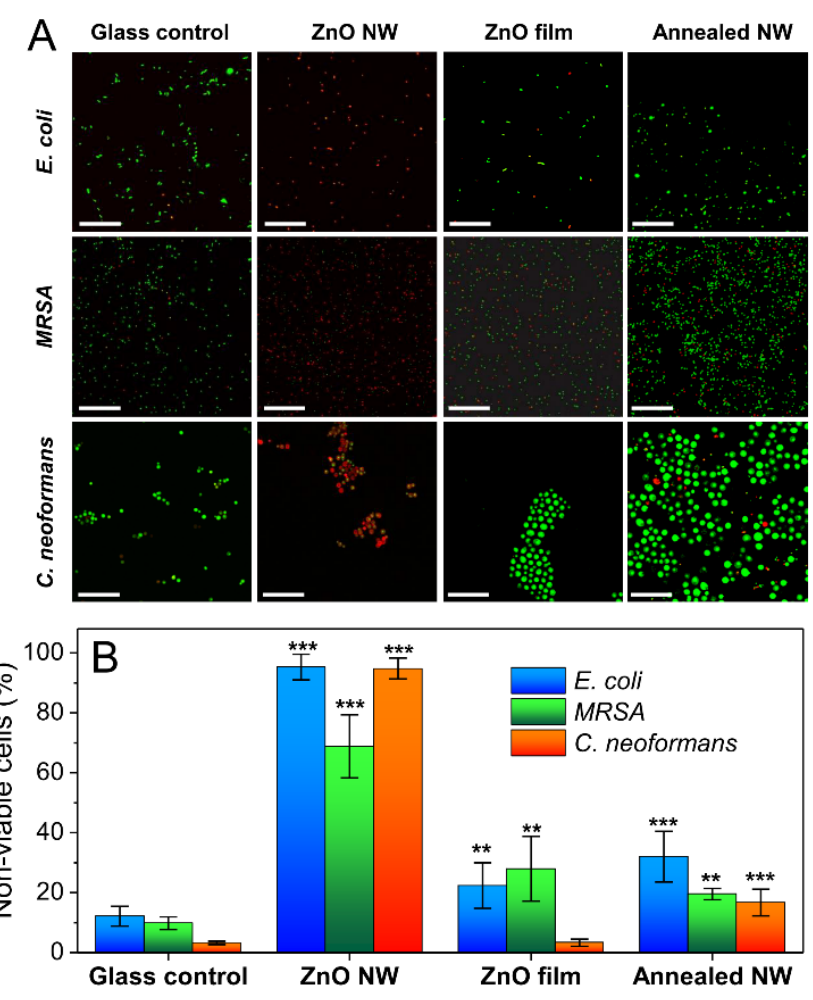

Figure 3. Cell viability studies. A) CLSM images of control (glass slides), $\mathrm{ZnO} \mathrm{NWs,} \mathrm{ZnO}$ dense film and annealed $\mathrm{ZnO} \mathrm{NWs}$ following immersion in E. coli, MRSA and C. neoformans for 24 
hours. After staining, the cells appear green (live) or red (dead) in the CLSM images. B) Quantification of cell viability obtained from the CLSM images. The statistical significance of the analysis as calculated by $\mathrm{t}$-tests against the data for the glass controls is reported as $*(\mathrm{p}<0.05),{ }^{* *}$ $(\mathrm{p}<0.01)$ or $* * *(\mathrm{p}<0.001)$.

These results highlight that the oxygen-deficient $\mathrm{ZnO} N W$ s exhibited a greater degree of lethality to all microbial species investigated, when compared to either the oxygen-deficient flat $\mathrm{ZnO}$, or the more stoichiometric NWs. This indicates that ZnO-based materials have inherent antibacterial properties, but that this activity is greatly enhanced by the presence of high-aspect-ratio nanostructures. Similarly, the mechanical (physical) action of the ZnO NWs is less effective in isolation, than when combined with a chemically active surface. This is noteworthy for two reasons. First, it highlights how chemical (oxygen vacancies) and physical (nanostructuring) features have a synergistic effect when combined, and greatly enhance the antimicrobial properties of $\mathrm{ZnO}$. Second, it demonstrates that $\mathrm{ZnO}$ NWs exhibit high activity for both bacterial and fungal cells. Since these cells have vastly different physiologies, this often precludes equivalent antimicrobial treatment. Indeed, fungal cells $C$. neoformans are typically more difficult to kill than E. coli, especially since this fungal species is resistant to the antifungal agent fluconazole. ${ }^{12}$ In light of this, the oxygen-deficient $\mathrm{ZnO}$ NWs show great promise as antimicrobial surfaces for both bacteria and fungi.

Interestingly, the antibacterial action of the NW surfaces is slightly less effective towards Grampositive MRSA cells. This effect has been shown previously and it has been ascribed to the different size and membrane rigidity of Gram-positive and Gram-negative bacteria. ${ }^{38,39}$ Specifically, MRSA cells are spherical, and on average smaller than E. coli, and therefore they 
interact with fewer NWs. Moreover, their cell membrane is more rigid compared to that of E. coli, which makes them more resilient to membrane stretching and tearing upon adsorption on textured surfaces. Compared to bacterial cells, fungal cells are often reported to possess thicker, more rigid cell walls, resulting in an overall stiffer membrane. ${ }^{12}$ However, due to their much larger size, they interact with a larger number of NWs, which explains the high activity of $\mathrm{ZnO}$ towards $C$. neoformans cells despite their resilient membrane and cell wall.

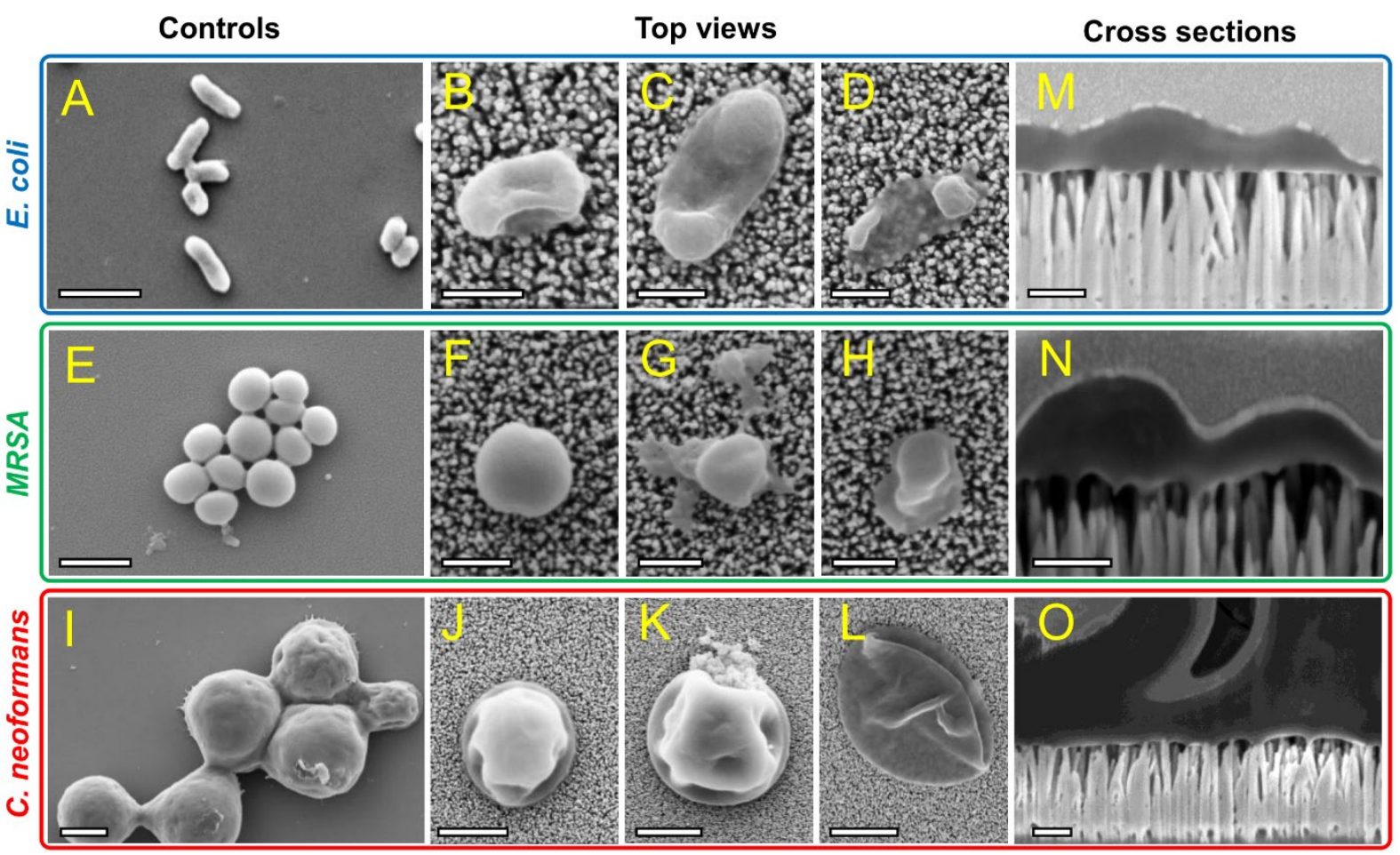

Figure 4. SEM characterization of the interaction between cells and $\mathrm{ZnO}$ nanowires. SEM images in top view (A-L) and cross section after FIB cutting (M-O) of cell interaction with control substrates (A,E,I) and ZnO NWs (all others). Scale bars for control samples are $2 \mu \mathrm{m}$ (A, I) and 1 $\mu \mathrm{m}$ (E). Scale bars for top views of ZnO NWs are $500 \mathrm{~nm}$ for (B-D and F-H) and $2 \mu \mathrm{m}$ for (J-L). Scale bars for cross sections are $200 \mathrm{~nm}$. As a point of interest, the nucleal cavity of the fungal cell can be clearly observed. 
Physical features (membrane rigidity, size) alone cannot explain the antimicrobial activity of the oxygen-deficient $\mathrm{ZnO} \mathrm{NWs}$, because annealed NWs show a drastically reduced activity, although still well above control levels. This is where the interplay between chemical and physical activity is vital. The chemical action of the $\mathrm{ZnO}$ surfaces is well-known, as $\mathrm{ZnO}$-based nanomaterials have been previously investigated as microbial inhibitory agents against both bacterial ${ }^{40}$ and fungal cells. ${ }^{41}$ Importantly, it has also been demonstrated that $\mathrm{ZnO}$-based nanomaterials are capable of producing reactive oxygen species $\left(\mathrm{ROS}\right.$ such as $\bullet \mathrm{OH}, \cdot \mathrm{O}_{2}^{-}$, and $\mathrm{H}_{2} \mathrm{O}_{2}$ ) as a consequence of surface defects. ${ }^{41,42,43}$ These ROS are capable of inducing cell lysis, via membrane and intercellular damage through both cell-surface interaction and general cell oxidation. ${ }^{44,45}$ Considering the highly oxygen deficient (defective) nature of the as-deposited $\mathrm{ZnO} \mathrm{NWs,} \mathrm{it} \mathrm{is} \mathrm{therefore} \mathrm{reasonable}$ to assume that they can generate ROS more effectively, and consequently it is not surprising that their ability to cause cell lysis is greater compared to less defective, although morphologically identical surfaces. We also note that a change in stoichiometry might cause a change in surface charge between different surfaces, and this might affect cell adhesion and interaction with the membrane. Importantly, leaching of zinc cations following dissolution of $\mathrm{ZnO}$ in the nutrient broth could potentially explain the antimicrobial activity of our $\mathrm{ZnO}$. To test this hypothesis, we measured the amount of zinc ions released from the different $\mathrm{ZnO}$ surfaces following 24 hours of immersion in the nutrient broth (Table S3). Zinc ions are detected in the broth for all three surfaces, in slightly larger amount for the NWs samples, consistent with their higher surface area with respect to flat $\mathrm{ZnO}$. However, no difference in leaching between pristine and annealed NWs could be observed. Therefore, considering the dramatic reduction in antimicrobial activity of the annealed NWs, we can confidently exclude a major role of zinc cations in the antimicrobial activity of our surfaces. SEM was used to analyze the cell/surface interaction: from the top-down images 
on control glass surfaces (Figure 4) we can clearly observe the three cell types investigated, each with their distinctive morphologies: elongated E. coli, spherical MRSA and larger spherical $C$. neoformans. The remaining top view SEM images presented in Figure 4 show cell rupture and eventually cell collapse (moving left to right in the images) for all microbial species interacting with oxygen deficient $\mathrm{ZnO}$ NWs. Compared to healthy control cells, the cells attached to the $\mathrm{ZnO}$ NWs show a stretched and deflated (flattened) membrane, a clear indication of damage that led (or will lead) to cell death (see also Figure S5). Importantly, in the cross-sectional images (Figure 4, right panels) the $\mathrm{ZnO}$ NWs can be observed to have entered the intracellular space of all microbial species. This indicates that during the cellular adsorption, the nanowires are capable of "puncturing" the pathogenic cells, via a combined physical/chemical mechanism as described earlier.

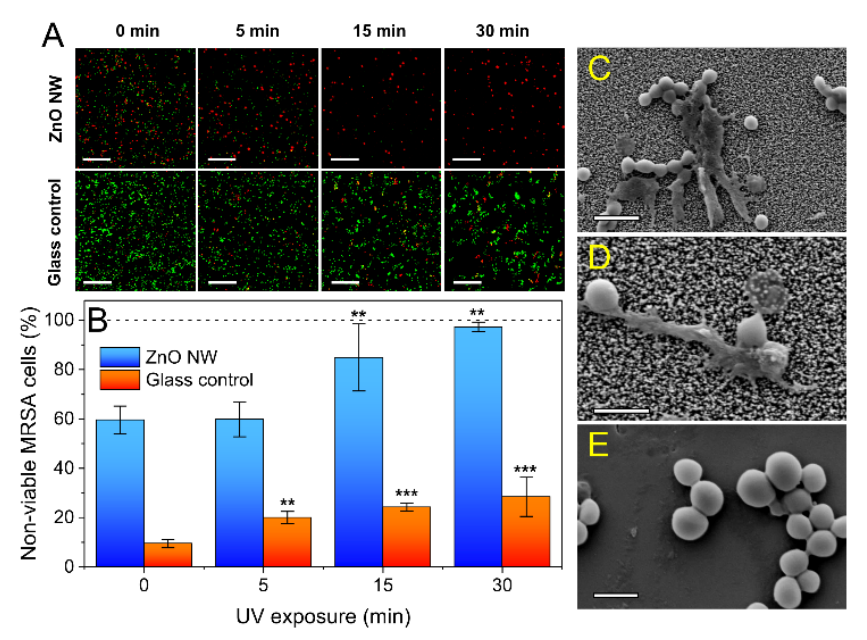

Figure 5. ZnO antibacterial properties under UV illumination. A) CLSM images of control (glass slides) and ZnO NWs following immersion in MRSA for 24 hours and exposed to UVA light for an increasing amount of time. B) Quantification of MRSA viability obtained from the CLSM images. The statistical significance of the analysis as calculated by t-tests against the samples without UV exposure $(0 \mathrm{~min})$ is reported as $*(\mathrm{p}<0.05), * *(\mathrm{p}<0.01)$ or $* * *(\mathrm{p}<0.001)$. C- 
E) SEM images of MRSA cells on $\mathrm{ZnO}$ NWs (C,D) and on glass (E) following exposure to UV light (30 min). Scale bars are $2 \mu \mathrm{m}$ in (C) and $1 \mu \mathrm{m}$ in (D, E).

In order to improve the activity of our $\mathrm{ZnO}$ NWs against the most resilient of the cell types tested (MRSA), we assessed their performance under UV irradiation. It is known that $\mathrm{ZnO}$ can be photodoped using ultraviolet light, which causes the photo-oxidation of adsorbates (including oxygen, water, carbon dioxide and organic contaminants) on the surface of $\mathrm{ZnO}^{46} \mathrm{We}$ postulate that the activation of the surface of our $\mathrm{ZnO}$ NWs could enhance their antimicrobial properties through the photo-induced oxidation of the adsorbed bacteria. To verify this, $\mathrm{ZnO}$ surfaces were exposed to low-energy UVA light using a commercial LED $\left(365 \mathrm{~nm}, 3 \mathrm{~mW} / \mathrm{cm}^{2}\right)$ after 24 hours of MRSA incubation and compared the results with glass controls which have be subjected to the same UV treatment. Illumination was done from the bottom of a Petri dish, therefore through the back of the substrate (glass). The power density of the UV light was measured on top of the glass slides through the Petri dish using a calibrated photodetector, and therefore is representative of the amount of UV light reaching the active surface. A progressive increase in activity for $\mathrm{ZnO}$ NWs with increasing UV exposure time is observed, which culminates with a striking 98\% MRSA cell death after 30 min of UV exposure (Figure 5). The values and associated errors are reported in the SI as Table S3. This data shows that the as-deposited $\mathrm{ZnO}$ NWs are highly antimicrobial even for MRSA, a notoriously resilient species, and these results provide a design rule for further improvements in efficacy for other antimicrobial surfaces and materials. We note that UV exposure slightly increase the antibacterial efficacy of other $\mathrm{ZnO}$ morphologies (flat films, Figure S6), however the results achieved by the ZnO NWs are still unparalleled. 
Importantly, only a small increase in the non-viable cell count is observed for the control glass slides after UV illumination. This increase might be due to a direct effect of UV light in damaging the MRSA cells. However, in the case of the $\mathrm{ZnO}$ NWs (as the samples are illuminated from the back side), over $90 \%$ of $\mathrm{UV}$ radiation is absorbed by $\mathrm{ZnO}$ before reaching the top surface and therefore the MRSA cells (see Figure S7). As a consequence, only less than $10 \%\left(\sim 0.3 \mathrm{~mW} / \mathrm{cm}^{2}\right.$, as measured using the photodetector) reaches the MRSA cells adsorbed on the $\mathrm{ZnO}$ surfaces, while the full $\mathrm{UV}$ power $\left(3 \mathrm{~mW} / \mathrm{cm}^{2}\right)$ is incident upon the cells in the glass control samples. Considering this, we can confidently conclude that it is the photodoping of $\mathrm{ZnO}$, and not the direct UV exposure, that causes the enhanced activity and close to $100 \%$ cell death in $\mathrm{ZnO}$ NWs samples. These results are extremely impressive as MRSA is notoriously very difficult to eradicate with high kill percentages.

\section{CONCLUSION}

In conclusion, we have shown the outstanding antimicrobial properties of oxygen-deficient $\mathrm{ZnO}$ nanowires towards bacteria (both Gram-positive and Gram-negative) and fungal cells. The work demonstrated that a combination of surface texturing and surface chemical environment is necessary to achieve the highest activity. Specifically, the antimicrobial activity of our optimized $\mathrm{ZnO}$ NW surfaces is reduced when either the texturing is lost, or the NWs are made less oxygen deficient. With our $\mathrm{ZnO}$ surfaces we achieved $\sim 95 \%$ antimicrobial activity for both E. coli (Gramnegative bacterium) and C. neoformans (fluconazole-resistant fungus). The activity was slightly lower (60-70\%) towards MRSA (Gram-positive bacterium), however it could be greatly improved to over $95 \%$ through a low intensity UVA exposure. These results highlight the synergistic effect between surface morphology and surface chemistry in order to achieve effective antimicrobial 
activity. Therefore, this study provides the foundation for further investigations into the combined physical and chemical activity of various (nano)materials and surfaces.

\section{METHODS}

\section{Materials}

Zinc acetate dihydrate (99\%), ethanolamine (99\%), 2-methoxyethanol (99\%), zinc nitrate hexahydrate (98\%), ammonium citrate dibasic (98\%) were purchased from Sigma Aldrich. Ammonium hydroxide solution (25\% in water) was purchased from Chem Supply. Acetone $(99.5 \%)$ and isopropanol (99.5\%) were supplied by Univar. Milli-Q water was used for all syntheses, and deionized water was used for all rinsing and washing steps. All chemicals were used without further purification. Standard microscope glass slides (soda-lime glass) were purchased from Livingstone. Silicon wafers (Boron doped, p-type, $<100>$ oriented, 10-20 $\Omega \mathrm{cm}$ ) were supplied by Silicon Materials.

\section{ZnO synthesis}

$\mathrm{ZnO}$ nanowires and films were synthesized using a modified chemical bath deposition (CBD) similar to previous reports. ${ }^{30}$ Glass and silicon substrates were cleaned via ultrasonication in acetone and isopropanol (IPA) for 10 minutes each, thoroughly rinsed with IPA and stored under IPA until needed. First, a ZnO sol-gel seed layer was deposited on glass and silicon substrates using a $0.4 \mathrm{M}$ solution of zinc acetate in 2-methoxyethanol, with a 1:1 molar ratio between zinc and ethanolamine. Films were deposited by spin coating at $2500 \mathrm{rpm}$ and annealed at $200{ }^{\circ} \mathrm{C}$ for 5 minutes. This procedure was repeated once more, with a final annealing at $200{ }^{\circ} \mathrm{C}$ for 30 minutes. Seeded substrates were then dipped in a $20 \mathrm{mM}$ zinc nitrate solution prepared in a $\sim 1 \%$ ammonium 
hydroxide solution and heated to $80{ }^{\circ} \mathrm{C}$ for 1 hour. Samples were positioned vertically, with the seeded surface facing slightly downwards. To obtain dense films, ammonium citrate was added to the growth solution with an overall concentration of $2 \mathrm{mM}$ right before dipping the seeded substrates. After 1 hour at $80^{\circ} \mathrm{C}$, samples were removed from the bath, rinsed thoroughly with water and dried with a nitrogen stream. The backside of the samples was wiped cleaned first with water, and then gently with a cotton bud dipped into a $\sim 0.1 \mathrm{M} \mathrm{HCl}$ solution to remove traces of precipitates that might have settled during deposition. Eventually samples were rinsed with water multiple times, dried thoroughly and stored under inert atmosphere until needed.

Bacterial and fungal strains, growth conditions, and sample preparation

Escherichia coli DH52 and methicillin-resistant Staphylococcus aureus ATCC1698 were obtained from the American Type Culture Collection (ATCC). Fluconazole-resistant Cryptococcus neoformans strains were obtained from a fluconazole resistant isolate derived from strain KN99 $\alpha$ which originally produced by Nielsen et al. ${ }^{47}$ For the bacterial experiments, bacteria cultures were grown on Luria-Bertani (LB) agar overnight at $37^{\circ} \mathrm{C}$. For fungal experiments, fungal culture was grown on yeast peptone dextrose (YPD) agar supplemented with $10 \%$ of glucose at $25{ }^{\circ} \mathrm{C}$. Bacterial and fungal cells were collected from the culture via an inoculation loop and suspended in LB and YPD (supplemented with 10\% glucose) broths. The density of the bacterial and fungal suspensions was then adjusted to $\mathrm{OD}_{600 \mathrm{~nm}}=0.1$.

\section{Characterizations}

$\mathrm{X}$-ray diffraction (XRD) patterns of $\mathrm{ZnO}$ nanowires and films deposited on glass were collected using a Bruker D4 Endeavor diffractometer equipped with a $\mathrm{Cu}-\mathrm{K} \alpha$ radiation source and operated at $40 \mathrm{kV}$ and $35 \mathrm{~mA}$. X-ray photoelectron spectroscopy (XPS) analyses were conducted on a 
Thermo Scientific K-Alpha XPS equipped with a Monochromated Al Ka X-ray source (1486.7 $\mathrm{eV}$ ) and a concentric hemispherical analyzer operated with a pass energy of $50 \mathrm{eV}$. Optical transmission spectra of $\mathrm{ZnO}$ films and nanowires deposited on glass substrates were obtained on an Agilent Cary 7000 UV-Vis-NIR spectrophotometer. Scanning electron microscopy (SEM) images of the $\mathrm{ZnO}$ prior to antimicrobial studies were acquired on a FEI Verios 460L SEM operated at $3-5 \mathrm{kV}$ and $25 \mathrm{pA}$. For top-view cellular imaging, all samples were affixed using formaldehyde/glutaraldehyde $3 \%$ solution in $0.1 \mathrm{M}$ sodium cacodylate buffer $(\mathrm{pH}=7.4)$, rinsed with PBS (10 mM, pH = 7.4), dehydrated using a series of 30\%, 50\%, 75\%, 90\% and $100 \%$ ethanol solutions, then air dried prior to coating with a thin film of gold and imaged using the same parameters described above. Cross sectional SEM of the $\mathrm{ZnO}$-cell interaction was performed using FEI Scios Dualbeam FIBSEM. The cells were first coated with platinum $(\mathrm{Pt})$ using an ion gun at $16 \mathrm{kV} / 0.15 \mathrm{nA}$ to prevent further damage followed by sequential ion slicing at $16 \mathrm{kV} / 0.15 \mathrm{nA}$. The imaging was then carried out at $2 \mathrm{kV}$ and $0.1 \mathrm{nA}$. Atomic force microscopy images were obtained using an Oxford Instrument Cypher ES AFM under ambient conditions. Olympus Micro Cantilevers (OMCL-AC240TS) with nominal spring constant $\mathrm{k}_{\mathrm{c}}=2.0 \mathrm{~N} / \mathrm{m}$ ) were used for all measurements, and imaging forces were minimized via a setpoint ratio (Imaging Amplitude (A)/free amplitude (A0)) of $>0.7$. Zinc leaching experiment was conducted by immersing $\mathrm{ZnO}$ samples in the nutrient broth for 24 hours and the broth was then analyzed with microwave plasma atomic emission spectroscopy (MP-AES) using an Agilent 4200 MP-AES instrument. Confocal scanning laser microscopy (CSLM) was performed on a Olympus Fluoview FV1200 inverted microscope and a ZEISS LSM 880 Airyscan upright microscope. Bacterial and fungal cells were dyed using a LIVE/DEAD BacLight Bacterial Viability Kit (including SYTO 9 and propidium iodide) and a LIVE/DEAD FungaLight Yeast Viability Kit, respectively (purchased from 
Invitrogen Molecular Probes). Cell dying was performed following an incubation period of 24 hours. The amount of live and dead cells was quantified using Cell-C software (https://sites.google.com/site/cellcsoftware/). The statistical significance of the analyses was calculated by t-tests against the control data and reported as $*(\mathrm{p}<0.05), * *(\mathrm{p}<0.01)$ or $* * *$ $(\mathrm{p}<0.001)$.

\section{ASSOCIATED CONTENT}

Supporting Information. Additional characterizations and data. This material is available free of charge via the Internet at http://pubs.acs.org

\section{AUTHOR INFORMATION}

\section{Corresponding Author}

*enrico.dellagaspera@rmit.edu.au (EDG),*vi.khanh.truong@rmit.edu.au (VKT)

\section{Author Contributions}

$\mathrm{AE}$ and EDG conceived the idea, directed the work and analyzed most of the data. PW and EDG synthesized the $\mathrm{ZnO}$ and conducted SEM and optical characterization. $\mathrm{AE}, \mathrm{SC}$ and VKT performed the antimicrobial studies, including SEM of the cell/surface interaction, with help from AEM and KJB. JK performed the XPS characterization. RJC, JC supervised the antimicrobial work. CFMcC and JvE helped with materials characterization and analysis. EDG and AE wrote the manuscript with inputs from all authors.

\section{ACKNOWLEDGMENT}

The Australian Research Council (ARC) is greatly acknowledged for supporting this work through the following grants: DE170100164 (EDG), DP190101864 (EDG). The Cypher ES AFM 
instrument was funded in part by grant LE170100096 from the ARC. The authors acknowledge

the facilities and the technical assistance of the RMIT University's Microscopy and Microanalysis

Facility (RMMF) and the MicroNano Research Facility (MNRF).

\section{REFERENCES}

1. $\quad$ Boucher, H. W.; Talbot, G. H.; Bradley, J. S.; Edwards, J. E.; Gilbert, D.; Rice, L. B.; Scheld, M.; Spellberg, B.; Bartlett, J., Bad Bugs, No Drugs: No ESKAPE! An Update from the Infectious Diseases Society of America. Clinical Infectious Diseases 2009, 48 (1), 1-12.

2. Levy, S. B.; Marshall, B., Antibacterial resistance worldwide: causes, challenges and responses. Nature Medicine 2004, 10, S122-S129.

3. $\quad$ Spellberg, B.; Guidos, R.; Gilbert, D.; Bradley, J.; Boucher, H. W.; Scheld, W. M.; Bartlett, J. G.; Edwards, J. J., The Epidemic of Antibiotic-Resistant Infections: A Call to Action for the Medical Community from the Infectious Diseases Society of America. Clinical Infectious Diseases 2008, 46 (2), 155-164.

4. Rodvold, K. A.; McConeghy, K. W., Methicillin-Resistant Staphylococcus aureus Therapy: Past, Present, and Future. Clinical Infectious Diseases 2014, 58 (suppl_1), S20-S27. 5. O'Neill, J., Tackling drug-resistant infections globally: Final report and recommendations. 2016. https://amr-review.org/ 2018.

6. Boyce, K.; Morrissey, O.; Idnurm, A.; Macreadie, I., Insights into the global emergence of antifungal drug resistance. Microbiology Australia 2019, 87-91.

7. Brown, G. D.; Denning, D. W.; Gow, N. A.; Levitz, S. M.; Netea, M. G.; White, T. C., Hidden killers: human fungal infections. Science translational medicine 2012, 4 (165), 165 rv13.

8. Knetsch, M. L. W.; Koole, L. H., New Strategies in the Development of Antimicrobial Coatings: The Example of Increasing Usage of Silver and Silver Nanoparticles. Polymers 2011, $3(1), 340$.

9. $\quad$ Kälicke, T.; Schierholz, J.; Schlegel, U.; Frangen, T. M.; Köller, M.; Printzen, G.; Seybold, D.; Klöckner, S.; Muhr, G.; Arens, S., Effect on infection resistance of a local antiseptic and antibiotic coating on osteosynthesis implants: An in vitro and in vivo study. Journal of Orthopaedic Research 2006, 24 (8), 1622-1640.

10. Nederberg, F.; Zhang, Y.; Tan, J. P. K.; Xu, K.; Wang, H.; Yang, C.; Gao, S.; Guo, X. D.; Fukushima, K.; Li, L.; Hedrick, J. L.; Yang, Y.-Y., Biodegradable nanostructures with selective lysis of microbial membranes. Nature Chemistry 2011, 3 (5), 409-414.

11. Banerjee, I.; Pangule, R. C.; Kane, R. S., Antifouling Coatings: Recent Developments in the Design of Surfaces That Prevent Fouling by Proteins, Bacteria, and Marine Organisms. Advanced Materials 2011, 23 (6), 690-718.

12. Elbourne, A.; Chapman, J.; Gelmi, A.; Cozzolino, D.; Crawford, R. J.; Khanh Truong, V., Bacterial-nanostructure interactions: the role of cell elasticity and adhesion forces. Journal of Colloid and Interface Science 2019, 546, 192-210.

13. Elbourne, A.; Truong, V. K.; Cheeseman, S.; Rajapaksha, P.; Gangadoo, S.; Chapman, J.; Crawford, R. J., The use of nanomaterials for the mitigation of pathogenic biofilm formation. In Methods in Microbiology, Gurtler, V.; Ball, A. S.; Soni, S., Eds. Academic Press: 2019; Vol. 46, pp 61-92. 
14. Bridier, A.; Briandet, R.; Thomas, V.; Dubois-Brissonnet, F., Resistance of bacterial biofilms to disinfectants: a review. Biofouling 2011, 27 (9), 1017-1032.

15. Johnston, H. J.; Hutchison, G.; Christensen, F. M.; Peters, S.; Hankin, S.; Stone, V., A review of the in vivo and in vitro toxicity of silver and gold particulates: particle attributes and biological mechanisms responsible for the observed toxicity. Critical reviews in toxicology 2010, 40 (4), 328-346.

16. Stigter, M.; Bezemer, J.; De Groot, K.; Layrolle, P., Incorporation of different antibiotics into carbonated hydroxyapatite coatings on titanium implants, release and antibiotic efficacy. Journal of controlled release 2004, 99 (1), 127-137.

17. Elbourne, A.; Crawford, R. J.; Ivanova, E. P., Nano-structured antimicrobial surfaces: From nature to synthetic analogues. Journal of Colloid and Interface Science 2017, 508 (Supplement C), 603-616.

18. Luong-Van, E.; Rodriguez, I.; Low, H. Y.; Elmouelhi, N.; Lowenhaupt, B.; Natarajan, S.; Lim, C. T.; Prajapati, R.; Vyakarnam, M.; Cooper, K., Review: Micro- and nanostructured surface engineering for biomedical applications. Journal of Materials Research 2012, 28 (2), 165-174.

19. Arias, A. C.; MacKenzie, J. D.; McCulloch, I.; Rivnay, J.; Salleo, A., Materials and Applications for Large Area Electronics: Solution-Based Approaches. Chemical Reviews 2010, $110(1), 3-24$.

20. Diodati, S.; Dolcet, P.; Casarin, M.; Gross, S., Pursuing the Crystallization of Monoand Polymetallic Nanosized Crystalline Inorganic Compounds by Low-Temperature WetChemistry and Colloidal Routes. Chemical Reviews 2015, 115 (20), 11449-11502.

21. Kim, H.-S.; Cook, J. B.; Lin, H.; Ko, Jesse S.; Tolbert, Sarah H.; Ozolins, V.; Dunn, B., Oxygen vacancies enhance pseudocapacitive charge storage properties of MoO3-x. Nature Materials 2016, 16, 454.

22. Elbourne, A.; Coyle, V. E.; Truong, V. K.; Sabri, Ylias M.; Kandjani, A. E.;

Bhargava, S. K.; Ivanova, E. P.; Crawford, R. J., Multi-directional electrodeposited gold nanospikes for antibacterial surface applications. Nanoscale Advances 2019, 1, 203-212.

23. Della Gaspera, E.; Guglielmi, M.; Agnoli, S.; Granozzi, G.; Post, M. L.; Bello, V.; Mattei, G.; Martucci, A., Au Nanoparticles in Nanocrystalline TiO2-NiO Films for SPR-Based, Selective H2S Gas Sensing. Chemistry of Materials 2010, 22 (11), 3407-3417.

24. Paglia, F.; Vak, D.; van Embden, J.; Chesman, A. S. R.; Martucci, A.; Jasieniak, J. J.; Della Gaspera, E., Photonic Sintering of Copper through the Controlled Reduction of Printed CuO Nanocrystals. ACS Applied Materials \& Interfaces 2015, 7 (45), 25473-25478.

25. van Embden, J.; Della Gaspera, E., Ultrathin Solar Absorber Layers of Silver Bismuth Sulfide from Molecular Precursors. ACS Applied Materials \& Interfaces 2019, 11 (18), 1667416682.

26. Luther, J. M.; Jain, P. K.; Ewers, T.; Alivisatos, A. P., Localized surface plasmon resonances arising from free carriers in doped quantum dots. Nature Materials 2011, 10, 361. 27. Della Gaspera, E.; Chesman, A. S. R.; van Embden, J.; Jasieniak, J. J., Non-injection Synthesis of Doped Zinc Oxide Plasmonic Nanocrystals. ACS Nano 2014, 8 (9), 9154-9163. 28. Mitzi, D. B.; Yuan, M.; Liu, W.; Kellock, A. J.; Chey, S. J.; Deline, V.; Schrott, A. G., A High-Efficiency Solution-Deposited Thin-Film Photovoltaic Device. Advanced Materials 2008, 20 (19), 3657-3662. 
29. Della Gaspera, E.; Griggs, J.; Ahmed, T.; Walia, S.; Mayes, E. L. H.; Calzolari, A.; Catellani, A.; van Embden, J., Augmented band gap tunability in indium-doped zinc sulfide nanocrystals. Nanoscale 2019, 11 (7), 3154-3163.

30. Della Gaspera, E.; Kennedy, D. F.; van Embden, J.; Chesman, A. S. R.; Gengenbach, T. R.; Weber, K.; Jasieniak, J. J., Flash-Assisted Processing of Highly Conductive Zinc Oxide Electrodes from Water. Advanced Functional Materials 2015, 25 (47), 7263-7271.

31. Guo, H.-L.; Zhu, Q.; Wu, X.-L.; Jiang, Y.-F.; Xie, X.; Xu, A.-W., Oxygen deficient ZnO1-x nanosheets with high visible light photocatalytic activity. Nanoscale 2015, 7 (16), 72167223.

32. Geng, Z.; Kong, X.; Chen, W.; Su, H.; Liu, Y.; Cai, F.; Wang, G.; Zeng, J., Oxygen Vacancies in $\mathrm{ZnO}$ Nanosheets Enhance CO2 Electrochemical Reduction to CO. Angewandte Chemie International Edition 2018, 57 (21), 6054-6059.

33. Singh, M.; Jampaiah, D.; Kandjani, A. E.; Sabri, Y. M.; Della Gaspera, E.; Reineck, P.; Judd, M.; Langley, J.; Cox, N.; van Embden, J.; Mayes, E. L. H.; Gibson, B. C.; Bhargava, S. K.; Ramanathan, R.; Bansal, V., Oxygen-deficient photostable $\mathrm{Cu} 2 \mathrm{O}$ for enhanced visible light photocatalytic activity. Nanoscale 2018, 10 (13), 6039-6050.

34. Tian, Z. R.; Voigt, J. A.; Liu, J.; McKenzie, B.; McDermott, M. J.; Rodriguez, M. A.; Konishi, H.; Xu, H., Complex and oriented ZnO nanostructures. Nature Materials 2003, 2 (12), 821-826.

35. Marrani, A. G.; Caprioli, F.; Boccia, A.; Zanoni, R.; Decker, F., Electrochemically deposited $\mathrm{ZnO}$ films: an XPS study on the evolution of their surface hydroxide and defect composition upon thermal annealing. Journal of Solid State Electrochemistry 2014, 18 (2), 505 513.

36. Della Gaspera, E.; Duffy, N. W.; van Embden, J.; Waddington, L.; Bourgeois, L.; Jasieniak, J. J.; Chesman, A. S. R., Plasmonic Ge-doped ZnO nanocrystals. Chemical Communications 2015, 51 (62), 12369-12372.

37. Della Gaspera, E.; Bersani, M.; Cittadini, M.; Guglielmi, M.; Pagani, D.; Noriega, R.; Mehra, S.; Salleo, A.; Martucci, A., Low-Temperature Processed Ga-Doped ZnO Coatings from Colloidal Inks. Journal of the American Chemical Society 2013, 135 (9), 3439-3448.

38. Xue, F.; Liu, J.; Guo, L.; Zhang, L.; Li, Q., Theoretical study on the bactericidal nature of nanopatterned surfaces. Journal of Theoretical Biology 2015, 385, 1-7.

39. Pogodin, S.; Hasan, J.; Baulin, Vladimir A.; Webb, Hayden K.; Truong, Vi K.; Phong Nguyen, The H.; Boshkovikj, V.; Fluke, Christopher J.; Watson, Gregory S.; Watson, Jolanta A.; Crawford, Russell J.; Ivanova, Elena P., Biophysical Model of Bacterial Cell Interactions with Nanopatterned Cicada Wing Surfaces. Biophysical Journal 2013, 104 (4), 835840 .

40. Jin, T.; Sun, D.; Su, J.; Zhang, H.; Sue, H. J., Antimicrobial efficacy of zinc oxide quantum dots against Listeria monocytogenes, Salmonella enteritidis, and Escherichia coli O157: H7. Journal of food science 2009, 74 (1), M46-M52.

41. Lipovsky, A.; Nitzan, Y.; Gedanken, A.; Lubart, R., Antifungal activity of ZnO nanoparticles - the role of ROS mediated cell injury. Nanotechnology 2011, 22 (10), 105101. 42. Dwivedi, S.; Wahab, R.; Khan, F.; Mishra, Y. K.; Musarrat, J.; Al-Khedhairy, A. A., Reactive Oxygen Species Mediated Bacterial Biofilm Inhibition via Zinc Oxide Nanoparticles and Their Statistical Determination. PLOS ONE 2014, 9 (11), e111289. 
43. Lakshmi Prasanna, V.; Vijayaraghavan, R., Insight into the Mechanism of Antibacterial Activity of ZnO: Surface Defects Mediated Reactive Oxygen Species Even in the Dark. Langmuir 2015, 31 (33), 9155-9162.

44. Sirelkhatim, A.; Mahmud, S.; Seeni, A.; Kaus, N. H. M.; Ann, L. C.; Bakhori, S. K. M.; Hasan, H.; Mohamad, D., Review on Zinc Oxide Nanoparticles: Antibacterial Activity and Toxicity Mechanism. Nano-Micro Letters 2015, 7 (3), 219-242.

45. Ranjan, S.; Ramalingam, C., Titanium dioxide nanoparticles induce bacterial membrane rupture by reactive oxygen species generation. Environmental Chemistry Letters 2016, 14 (4), 487-494.

46. Morfa, A. J.; MacDonald, B. I.; Subbiah, J.; Jasieniak, J. J., Understanding the chemical origin of improved thin-film device performance from photodoped $\mathrm{ZnO}$ nanoparticles. Solar Energy Materials and Solar Cells 2014, 124, 211-216.

47. Nielsen, K.; Cox, G. M.; Wang, P.; Toffaletti, D. L.; Perfect, J. R.; Heitman, J., Sexual cycle of Cryptococcus neoformans var. grubii and virulence of congenic a and $\alpha$ isolates.

Infection and immunity 2003, 71 (9), 4831-4841. 